Supporting information for

\title{
Role of the coulombic interaction in ligand-induced biopolymer aggregation
}

\author{
Norberto Micali* ${ }^{1}$ Valentina Villari, ${ }^{1}$ Matteo Cusumano, ${ }^{2}$ \\ Maria Letizia Di Pietro, ${ }^{2}$ and Antonino Giannetto ${ }^{2}$ \\ ${ }^{1}$ CNR-Istituto per i Processi Chimico-Fisici, \\ Via La Farina 237, I-98123, Messina, Italy \\ ${ }^{2}$ Dipartimento di Chimica Inorganica, \\ Chimica Analitica e Chimica Fisica, Università di Messina, \\ C.da Papardo S.ta Sperone 31, I-98166, Messina, Italy
}

\footnotetext{
* corresponding author: Norberto Micali, Phone: +39 090 2939693, Fax: +39 090 2939902, e-mail: micali@me.cnr.it.
} 


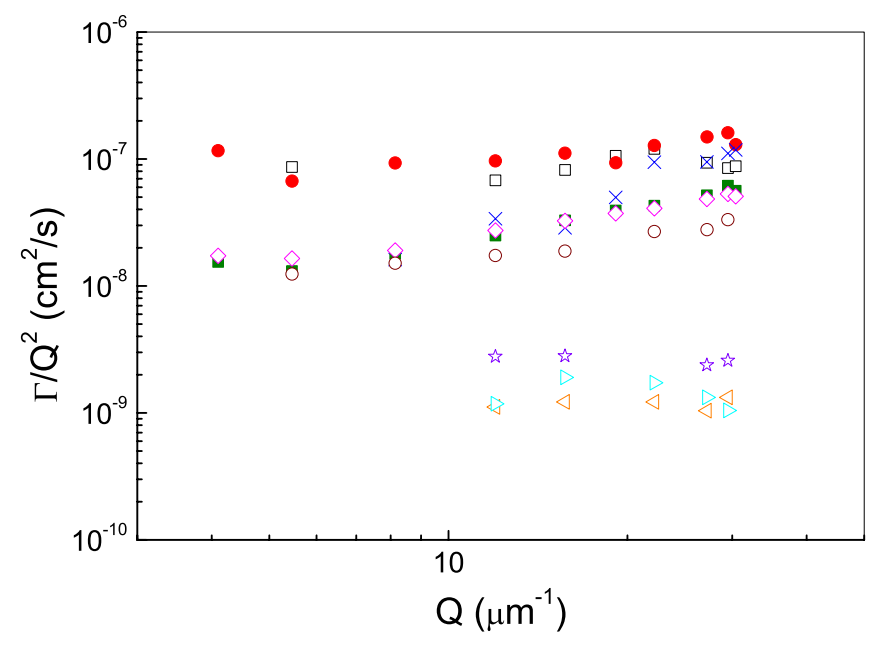

FIG. 1: Dependence of the relaxation rate on the exchanged wave vector for PolyA (hollow squares) and for $\mathrm{Pt}($ Terpy)/PolyA solutions (stabilized for four hours) at different molar ratios: 0.25 (filled circles), 0.4 (crosses), 0.5 (filled squares), 0.55 (diamonds), 0.6 (circles), 0.8 (left triangles), 0.9 (right triangles) and 1 (stars). 


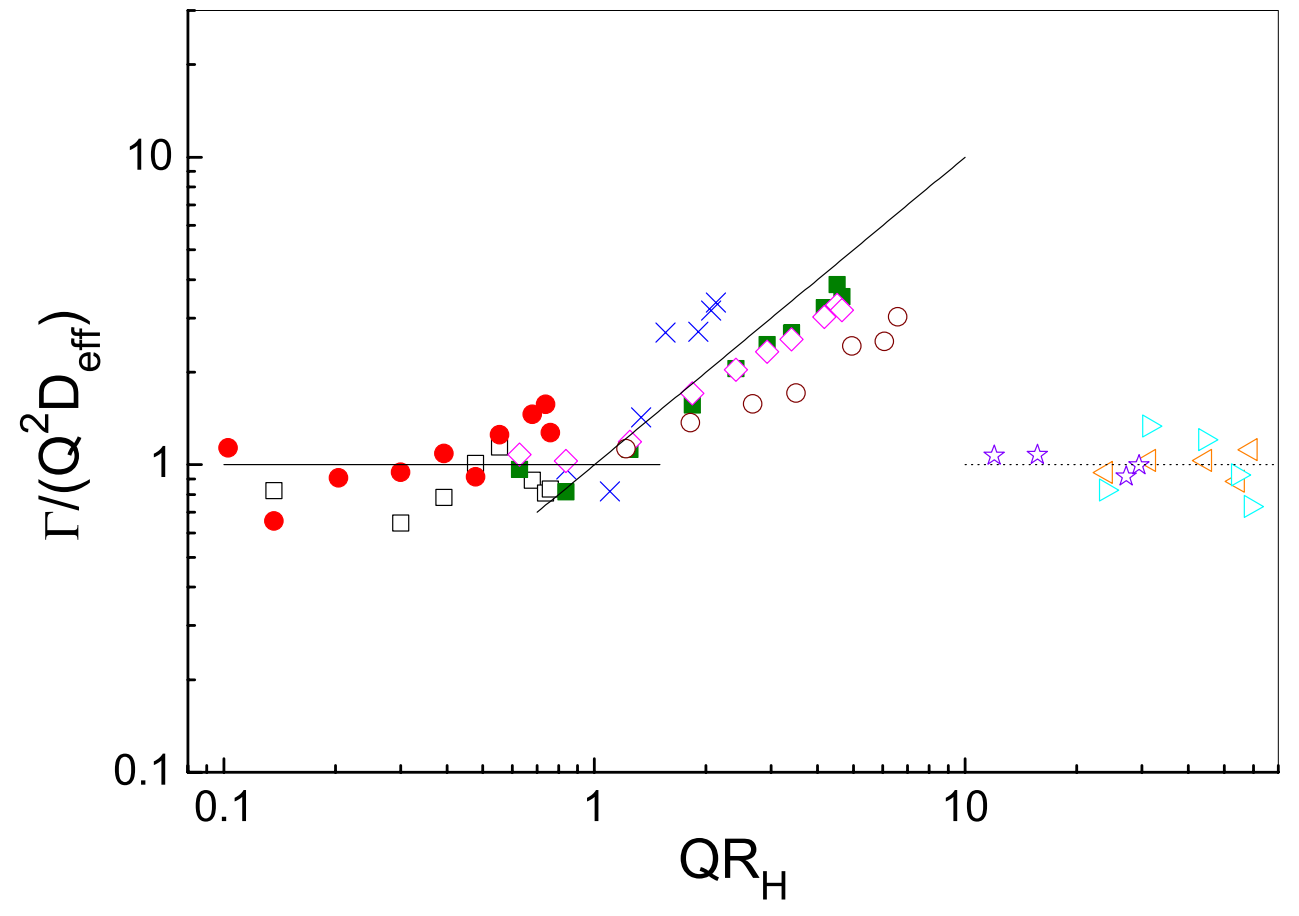

FIG. 2: Normalization of data in Fig.1 by the diffusion coefficient. The straight lines indicate the $Q^{2}$ and $Q^{3}$ regime. 